\title{
Interactive comment on "Atmospheric blocking types: Frequencies and transitions" by Carola Detring et al.
}

\section{Anonymous Referee \#2}

Received and published: 30 January 2021

Detring et al. present an analysis of long-term blocking changes over the past 30 years in the Northern Hemisphere. Using a novel method based on the kinematic vorticity number, the authors distinguish between long-term changes of Omega-blocks and High-over-low blocks. Though a general change of the blocking frequency over the 30 year period is not found, the authors identify pronounced changes when investigating trends on a monthly or seasonal basis. A key outcome is that Omega blocking is more likely to occur and to be more persistent than the High-over-Low blocking pattern.

To understand the changes/trends of blocking is an important topic in our science. Thus, the analysis is timely and the topic is likely to be of interest to the readership of WCD. However, there is a number of issues which need to be addressed before the manuscript can be published. 
Most important comments: 1) At many places trends/long-term changes are reported. However, most of the times, statements concerning the statistical significance of the results are missing. Further, the computation of the confidence intervals is not explained so that it remains unclear which of the results are "real" results. Investigating and commenting on the significance is particularly important since some of the results are likely to be based on small sample sizes. For example, it is reported that 13 (10) blocks occur per year in the large (small) domain. When breaking this down to a monthly basis the average number of blocks per month decreases to 1-2.

2) The trends inferred from the logistic regression approach are quite pronounced when considering individual months. For example, the logistic regression suggests that the probability of omega blocks during February has increased by a factor of three over the past 30 years. To better compare these results to the "observations" it would be beneficial if the actual numbers of blocked time steps were included in the corresponding figures. For example, vertical bars (in the same colours as the modeled probabilities) showing these numbers could be included in the panels of Fig. 8.

3) A focus of the study is on observed blocking trends. However, references to related studies are missing in the introduction (Section 1). To put the results in context to previous studies, I strongly encourage the authors to provide information on observed trends found in other studies. To avoid an excessively long introduction, the discussion of weather regimes and Grosswetterlagen could be shortened considerably.

4) The discussion in Section 5 is in large parts a repetition of Section 4. Many results are listed so that it is difficult for the reader to really identify the key outcomes of the study. In my view the discussion part would improve considerably if the authors formulated the key outcomes of the study more clearly and discussed their broader implications. For example, three key questions are raised in Section 1. Coming back to these questions in Section 5 would be one way to list the key results. Also, what are the broader implications of changing transition probabilities? Are some of these transitions particularly relevant for high impact weather or certain extremes? A further

Interactive comment 
comment on Section 5 is that several caveats of the methodology are mentioned but it remains unclear whether and in which way these caveats affect the results. Though WCDD I generally advocate such critical discussions, clearer statements regarding the effect on the results are mandatory.

Minor comments: Title: The title is very general. Please try to be more specific. For example, "Observed frequency and transition trends of Omega- and High-overlow blocks" would be a more specific title which might help to increase the visibility of the article.

I. 1: Consider to replace "very high" with "anomalously high". In some regions of the world "very high" may not mean "very high" in other regions of the world.

I. 2: l'd suggest to use the wording "flanked by" instead of "in combination with".

I. 6: here and elsewhere: Northern Hemisphere with capital letters.

I. 8: "temporal development" is quite unspecific. Do you mean the long-term change/trend of blocking probabilities?

I. 14: Initially and also later in the manuscript you talk about "weather patterns". Here, you introduce "weather regime". I suggest to use one of these terminologies consistently throughout the paper. In my opinion, "weather regime" is better suited than "weather pattern".

I. 24: Consider to write "steady ridges" in italic font since this is a third type of blocking.

I. 27: "can" instead of "could"

I. 27: Please provide a reference showing that these transition can be "often" observed.

I. 32: Please specify that this statement refers to central Europe (I guess).

I. 36: The Deutscher Wetterdienst invalidated the record of $42.6^{\circ} \mathrm{C}$ recently (https://www.dwd.de/DE/presse/pressemitteilungen/DE/2020/20201217_annulierung_lingen_news.html). 
Please adapt the manuscript accordingly.

I. 46: Please remove brackets around (Helmholtz, 1858).

WCDD

I. 47: Missing full stop after circulation.

I. 47: Why do you introduce the terminology "positive circulation" and "negative circulation"? This terminology is not used at all in the manuscript.

Interactive

comment

I. 83: Please use capital "S" when referring to a specific section (e.g., Section 2, Section 3, Section 3.2 etc).

I. 93: Please explain why you decided to focus on the period 1990 to 2019. The NCEP-DOE Reanalysis are available for the period 1979-2020. Why did you decide to not include the 1980s period for your analysis? Since you are looking at long-term changes, 10 years of additional data may help to make your statements more robust.

I. 93: Better write "close to zero" instead of "very low". "Very low" could also mean "negative".

I. 113: Better write "identify" or "capture" instead of "catch".

I. 120-122: Please clarify this explanation. Does this mean you disregard one block if two blocks occur at the same time in different parts of the area? Or do you simply assign the two blocks to the same blocking type (HoL vs Omega)?

I. 140: Do you employ any criteria concerning the minimum size of the vortex patches?

I. 142: I fully understand that you are not providing all details of Hirt et al. 2018. But could you at least mention the step size at which the size of the box increases? Is it $2.5^{\circ} ?$

I. 152: What exactly to you mean by "below the high center"? Do you mean south/equatorwards of the high center?

Printer-friendly version

I. 158: Replace "where we" by "who". 
I. 160: What exactly do you mean by "large jumps"? Please be more specific and provide the threshold in $\mathrm{km}$ or degree longitude.

I. 157: How are the "circulation centroids" in Fig. 3b identified? This needs to be explained in the text.

I. 165: The information on the life time is redundant and could be removed from the

Interactive manuscript.

I. 168: Please use consistent terminology for the "Euro-Atlantic sector" (here you are using "European sector" elsewhere it is "Euro-Atlantic sector").

I. 219: Better provide a reference to the work of Andrey Markov.

I. 248: Remove brackets around "e.g., Baclawski, 2008".

I. 256: Do you mean "seasons" instead of "Seas"? Or did I miss the definition of this acronym?

I. 271: Are you really displaying a frequency in Fig. 5 ? Or is it rather the number of events in the 30 -year period?

I. 279: Please avoid descriptive information which can also be derived from the figure caption.

I. 293: Please insert "a" between "of" and "blocking".

I. 300: I could not find the "straight-line estimates" in Section 4.1.2. My suggestion would be to include the straight line estimates in Fig. 6 (also for the benefit of the discussion in Section 4.1.2).

I. 300: Please see my comment on line 279.

Printer-friendly version

I. 302: Insert "The" before "Average".

I. Please be cautious concerning the terminology of "probability". Sometimes probability is used to describe a fraction (e.g., $301 p=0.24$ ), here probability is used to describe 
a percentage. l'd suggest to be consistent regarding the terminology.

I. 305: Please include references to the corresponding subfigures (Fig. 7a, b and so on).

I. 314: In Section 4.1.2 on average 109.8 blocks are found per year. When describing blocking probabilities on a monthly basis, did you not encounter any issues regarding the small sample size. Apologies if I missed this information.

comment

I. 321: Use "For example" instead of "E.g." at the beginning of a sentence.

I. 332: l'd suggest to write "...that September to March are characterized...". It is a bit odd to include "September" in the winter season.

I. 335: Clarify that it is not the "Euro-Atlantic" region which peaks in April, but the blocking frequency which peaks in April.

I. 346: Please remove "the" before "both".

I. 354: Please include "the" before "probability".

I. 359: "a" instead of "an".

I. 377: The terminology "offset" is a bit awkward. Please reconsider this terminology. The term "decay" is used more frequently to describe the transition from blocking to no blocking.

I. 390: Why is the change in transition probabilities of particular interest? Are these associated with particular weather phenomena so that any long-term changes would have an immediate impact?

I. 394: What about the persistence? According to the bottom right figure, the persistence of blocking has decreased which would indicate shorter blocking durations. In Section 4.1.2 however, a slight increase in blocking duration is reported. Can you comment on this contradiction?

Printer-friendly version

Discussion paper 
I. 394: Please label subfigures with a), b), c) etc. If subfigures are not discussed in the text (e.g., top left and bottom right in Fig. 13) this can be deleted from the manuscript.

I. 396-400: Are these conclusions only valid for JJA or is it for all seasons? Please clarify in the manuscript.

I. 396: A legend is missing in Fig. 14. This makes it hard to follow the discussion.

Interactive

comment

I. 404: The subfigures need to be labeled and referenced in the text. Otherwise, it is difficult to follow the discussion. Please consider to delete subfigures from the manuscript which are not discussed in the paper.

I. 421-430: This paragraph basically only tells the reader what has been done and is thus a repetition of Section 4 . Unless the authors have a strong argument on why this paragraph is important, I strongly recommend this paragraph from the manuscript.

I. 435: Where is it shown that the increase can be attributed to blocking events that occur over western Russia? Either show a Figure or reference other studies which support this interpretation.

I. 442: Replace "E.g." with "For example".

I. 443: Can you comment on the discrepancy between the study by Brunner et al. (2017) and this study? According to Fig. 8, the probability of blocking in February has increased by a factor of three (0.08 to 0.24$)$. Do you have any explanation on why the conclusion in Brunner et al. (2017) is completely different?

I. 459: Can you be more specific about this statement? Does "deviations" mean that the trends reverse when using different parameters?

I. 498: In my opinion you could easily find out whether your results coincide with the results of Drouard and Wollings (2018). For example, you could display the occurrence frequency of High-over-Low and Omega blocks as a function of longitude. Though I do not expect such a figure in the manuscript, I would be very interested to see such an

Printer-friendly version

Discussion paper 
analysis to better put this study in context with previous studies.

I. 510-516: How do these results relate to previous studies? Are these in line with WCDD previous results or do they contradict earlier studies?

I. 518: A verb is missing in this sentence.

I. 521: This sentence needs clarification. As it stands now "an increase in overall prob-

Interactive

comment ability for blocking" and "a higher proportion towards the end of the study" somewhat mean the same thing. I guess you want to say that the number of all blocks increases and that the fraction of Omega blocks increases, too.

I. 525: Replace "this" with "these".

Interactive comment on Weather Clim. Dynam. Discuss., https://doi.org/10.5194/wcd-2020-62, 2020. 